\title{
Link between Short Sleep Duration and Hypertension
}

\author{
Ki Dong Ko* \\ Department of Family Medicine, Gachon University Gil Medical Center, Incheon, Korea
}

\section{To the Editor}

I read with great interest the article by Song et al., ${ }^{1)}$ titled "The association between sleep duration and hypertension in nonobese premenopausal women in Korea". The investigators showed the association between short sleep duration $(<6$ hours a night) and hypertension in non-obese premenopausal women, but not in obese premenopausal women. In contrast, long sleep duration ( $>8$ hours a night) was not associated with hypertension in either non-obese or obese women. ${ }^{1)}$

As this study provides valuable information, I would like to discuss a few points. First, this study may have an information bias that leads to the incorrect classification of variables. For example, sleep duration measures were self-reported. This study could be improved by more objective measures, such as actigraphy and polysomnography, to address subjective or false reports. Understandably, it is difficult to adopt objective measures in the Korean National Health and Nutrition Examination Survey because they are costly and inconvenient, and therefore, several previous studies have accepted the limitation of self-reports. On the contrary, this study is strengthened by the fact that the hypertension variable was based either on self-report of physician diagnosis or on two blood pressure readings. As hypertension frequently goes undiagnosed, using only self-report of physician diagnosis, as has been done in many other studies, can create misclassification bias. Second, studies investigating the relationship between sleep duration and hypertension include various other variables (age, sex, menopause, socioeconomic status, smoking, caffeine/alcohol consumption, obesity-related variables, cardiovascular diseases, depression, snoring, or sleep apnea) and consider them as either potential confounders or mediators of the relationship. In my opinion, obesity should be regarded as a partial mediator between short sleep duration and hypertension. Therefore, stratification and additive adjustment in model 3 for obesity in this study might result in attenuation away from the true association (that is, over-adjustment). ${ }^{2)}$ Third, it was found in this study that long sleep duration was not associated with hypertension in study subjects. To the best of my knowledge, no published longitudinal studies have shown the association between long sleep duration and hypertension, although some cross-sectional studies have shown this. ${ }^{3-5)}$ In fact, there is little biological plausibility that long sleep duration itself has adverse health effects. ${ }^{6}$ Instead, those with hypertension may have elevated proinflammatory cytokines, which presumably induce sleepiness and fatigue., ${ }^{7,8)}$ Considering this, it is likely that long sleep duration is an epiphenomenon of hypertension rather than a cause. Fourth, this study was conducted among premenopausal women with a mean age of 35.5 years old. Interestingly, many epidemiological studies have indicated that the relationship between short sleep duration and hypertension is stronger in female subjects. ${ }^{9-12)}$ Cappuccio et al. ${ }^{9)}$ in the Whitehall II study pointed out a few potential mechanisms, including true biological interaction between short sleep duration and sex, differential self-reporting of sleep duration, and distribution differences of correlates of short sleep duration (for example, incidence of depression may be higher in women reporting short sleep duration). Moreover, study subjects restricted to premenopausal and relatively younger women may heighten the strength of association between short sleep duration and hypertension, compared to postmenopausal women who already have major hormonal changes and psychosocial stress. ${ }^{11,12)}$ Accordingly, the selection of subjects in this study does make sense.

Despite inconsistent results in subpopulations, there are lines of evidence from experimental sleep deprivation stud- 
ies $^{13)}$ and population-based epidemiological studies ${ }^{3-5,9-12)}$ suggesting that short sleep duration is linked with hypertension. A recent metaanalysis of longitudinal studies also found short sleep duration to increase the risk of hypertension. ${ }^{14)}$ Sleep restriction can activate several biological pathways, such as augmented activity of the sympathetic nervous system, altered hormone levels (elevated cortisol, elevated ghrelin, and reduced leptin), increased oxidative stress/inflammation, and accelerated atherosclerosis. ${ }^{13)}$ All of these changes are thought to be responsible for the link between short sleep duration and hypertension. ${ }^{13)}$

In conclusion, there is sufficient evidence that short sleep duration acts as a risk factor for hypertension. Thus, primary care providers should pay attention to early detection and prevention of hypertension in subjects with sleep deprivation.

\section{CONFLICT OF INTEREST}

No potential conflict of interest relevant to this article was reported.

\section{REFERENCES}

1. Song MY, Sung E, Jung SP, Lee KM, Keum SH, Ryu SD. The association between sleep duration and hypertension in non-obese premenopausal women in Korea. Korean J Fam Med 2016;37:130-4.

2. Jiang T, Baxter P, Tilling K, Ellison G, Gilthorpe M. PP17 Common statistical errors: over-/under-adjustment for mediators and confounders in lifecourse research. J Epidemiol Community Health 2014;68(Suppl 1):A53.

3. Gottlieb DJ, Redline S, Nieto FJ, Baldwin CM, Newman AB, Resnick $\mathrm{HE}$, et al. Association of usual sleep duration with hypertension: the Sleep Heart Health Study. Sleep 2006;29:1009-14.

4. Magee CA, Kritharides L, Attia J, McElduff P, Banks E. Short and long sleep duration are associated with prevalent cardiovascular disease in
Australian adults. J Sleep Res 2012;21:441-7.

5. Gangwisch JE, Feskanich D, Malaspina D, Shen S, Forman JP. Sleep duration and risk for hypertension in women: results from the nurses' health study. Am J Hypertens 2013;26:903-11.

6. Knutson KL, Turek FW. The U-shaped association between sleep and health: the 2 peaks do not mean the same thing. Sleep 2006;29:878-9.

7. Stumpf C, John S, Jukic J, Yilmaz A, Raaz D, Schmieder RE, et al. Enhanced levels of platelet P-selectin and circulating cytokines in young patients with mild arterial hypertension. J Hypertens 2005;23:9951000.

8. Krueger JM, Obal FJ, Fang J, Kubota T, Taishi P. The role of cytokines in physiological sleep regulation. Ann N Y Acad Sci 2001;933:211-21.

9. Cappuccio FP, Stranges S, Kandala NB, Miller MA, Taggart FM, Kumari M, et al. Gender-specific associations of short sleep duration with prevalent and incident hypertension: the Whitehall II Study. Hypertension 2007;50:693-700.

10. Stang A, Moebus S, Mohlenkamp S, Erbel R, Jockel KH; Heinz Nixdorf Recall Study Investigative Group. Gender-specific associations of short sleep duration with prevalent hypertension. Hypertension 2008; 51:e15-6.

11. Stranges S, Dorn JM, Cappuccio FP, Donahue RP, Rafalson LB, Hovey $\mathrm{KM}$, et al. A population-based study of reduced sleep duration and hypertension: the strongest association may be in premenopausal women. J Hypertens 2010;28:896-902.

12. Kim SJ, Lee SK, Kim SH, Yun CH, Kim JH, Thomas RJ, et al. Genetic association of short sleep duration with hypertension incidence: a 6-year follow-up in the Korean genome and epidemiology study. Circ J 2012;76:907-13

13. Tobaldini E, Costantino G, Solbiati M, Cogliati C, Kara T, Nobili L, et al. Sleep, sleep deprivation, autonomic nervous system and cardiovascular diseases. Neurosci Biobehav Rev 2017;74(Pt B):321-9.

14. Meng L, Zheng Y, Hui R. The relationship of sleep duration and insomnia to risk of hypertension incidence: a meta-analysis of prospective cohort studies. Hypertens Res 2013;36:985-95. 